\title{
Seseo, ceceo y distinción en el sociolecto alto de la ciudad de Sevilla: nuevos datos a partir de los materiales de PRESEEA
}

\author{
Juana Santana Marrero ${ }^{1}$ \\ Universidad de Sevilla
}

\begin{abstract}
Resumen
Los fonemas $/ \mathrm{s} / \mathrm{y} / \theta /$ en el ataque silábico presentan una gran heterogeneidad de realizaciones en el español hablado en Andalucía. Las investigaciones sociolingüísticas llevadas a cabo hasta el momento sobre zonas urbanas demuestran que su distribución está condicionada por los rasgos sociales de los hablantes: edad, sexo y nivel sociocultural. En lo que respecta a la ciudad de Sevilla, los estudios realizados sobre grabaciones de los años setenta reflejaban una tendencia mayoritaria a seguir el modelo de prestigio local seseante, en convivencia con la distinción s/ $\theta$, esta última con menor representatividad. El análisis que aquí se desarrolla toma como punto de partida los materiales de sociolecto alto de PRESEEA-Sevilla, que incluyen grabaciones recientes de encuestas semi-dirigidas realizadas entre los años 2009 y 2015. Los resultados alcanzados revelan el avance de la solución distinguidora s $/ \theta$, principalmente en la primera generación y en las mujeres, en detrimento de la variante vernácula seseante. Los índices de vacilación entre las dos pronunciaciones
\end{abstract}

\footnotetext{
$1 \quad$ Para correspondencia, dirigirse a: Juana Santana Marrero (jsanata@us.es), Facultad de Filología, Departamento de Lengua Española, Lingüística y Teoría de la Literatura, Universidad de Sevilla, C/ Palos de la Frontera, s/n 41004 - Sevilla, España.
} 
en un mismo informante se dieron mayoritariamente en la tercera generación. Se estudian, además, tres factores que pueden haber influido en la elección de una u otra opción: el entorno en el que aparecen los fonemas (simple o doble), la repetición de una misma lexía y su uso en sustantivos propios.

Palabras clave: pronunciación, Sevilla, sociolecto alto, sociolingüística, PRESEEA.

\title{
Seseo, ceceo and distinction in the high sociolect of Sevilla CITY: NEW DATA FROM PRESEEA
}

\begin{abstract}
The phonemes $/ \mathrm{s} /$ and $/ \theta /$ at the beginning of syllable position present a wide diversity of performances in the Spanish spoken in Andalusia. Sociolinguistic researches conducted so far on urban areas show that such distribution is conditioned by the social characteristics of speakers: age, sex and socio-cultural level. With respect to Seville city, previous studies on recordings of the seventies showed a tendency to follow the local prestige seseante model in coexistence with distinction $\mathrm{s} / \theta$, this one less representative. The analysis developed here is based on PRESEEA materials from Sevillian high sociolect, including recent recordings of semi-structured surveys recollected between 2009 and 2015. The results achieved reveal the progress of etymological solution, mainly in the first generation and females, at the expense of vernacular variant seseante. Rates of hesitation between the two pronunciations in one speaker were mainly found in the third generation. It is also studied three factors that may have influenced the choice of one or another: the immediate context in which the phonemes appear (single or double), the repetition of the same word and their occurrence inside proper nouns.
\end{abstract}

Keywords: Pronunciation, Seville, high sociolect, Sociolinguistics, PRESEEA.

Recibido: 13/05/2016

Aceptado: 01/07/2016 


\section{INTRODUCCIÓN}

Los fonemas /s/y / $/$ / en posición inicial de sílaba presentan varias soluciones en el español hablado en Andalucía: mantenimiento y neutralización de la oposición fonológica, esta última, a su vez, con reducción a /s/, el llamado seseo, y a / / /, el denominado ceceo (Navarro Tomás et al. 1933; Narbona et al. 1998: 131; Jiménez 1999: 31-32). Desde el punto de vista de la variación quedan implicadas dos variables fonológicas y sus correspondientes variantes: entre otras, mantenimiento de /s/ en [s] y solución ceceante en $[\theta] ; \mathrm{y}$ mantenimiento de $/ \theta /$ en $[\theta]$ y solución seseante [s]. La situación se complica si tenemos en cuenta que en la variedad andaluza estas realizaciones no se reparten de forma homogénea. Muy al contrario, presentan un panorama multiforme en el que alternan más de una de estas realizaciones fonéticas en un área geográfica, dentro de un grupo social o en un solo hablante (dependiendo de la situación comunicativa o, incluso, en un mismo contexto de habla).

En este trabajo nos ocupamos de describir la rentabilidad de los fenómenos de seseo, ceceo y distinción s/ $\theta$ en el sociolecto alto de la ciudad de Sevilla. Concretamente, estudiaremos si, según se ha puesto de manifiesto en áreas urbanas como Granada (Salvador 1980; Moya Corral y García Wiedemann 1995; Moya Corral y Sosiński 2015), Málaga (Villena 1997) o en cierta medida Jerez (García-Amaya 2008), se documentan procesos de convergencia hacia el modelo septentrional (que en nuestro caso quedaría reflejado por el mantenimiento de la distinción fonológica); o si se aprecian mecanismos de divergencia hacia los rasgos dialectales (no mantenimiento de la oposición, bien sea con solución seseante o con solución ceceante), según se ha observado en las ciudades de Córdoba (Uruburu 1996) y de Sevilla (Lamíquiz y Carbonero 1987). Será preciso analizar también si dichos fenómenos están influidos por la edad y el sexo de los hablantes. Además, tendremos ocasión de comparar nuestros resultados con los obtenidos en investigaciones previas realizadas en Sevilla capital (Carbonero 1982, 2003a [1985] y 2003b [2000]; Lamíquiz y Carbonero 1987), con el fin de observar si se siguen manteniendo las mismas tendencias de décadas atrás o si se perciben procesos de cambio.

Para llevar a cabo esta investigación nos basamos en los materiales del Proyecto de Estudio Sociolingüistico del Español de España y América (PRESEEA) de la ciudad hispalense, recopilados por los miembros del grupo de investigación Sociolingüistica Andaluza: Estudio Sociolingüistico del Habla de Sevilla (HUM 141). Este trabajo se encuadra específicamente 
entre los objetivos del Proyecto Patrones Sociolingüísticos del Español de Sevilla $(P A S O S-S E)^{2}$.

\section{ACERCA DE LA DISTRIBUCIÓN DE SESEO, CECEO Y DISTINCIÓN EN ANDALUCÍA}

La bibliografía existente sobre estos fenómenos es abundante, en tanto que han sido abordados en diversas ocasiones desde diferentes puntos de vista. Si nos atenemos a los datos de la distribución espacial podemos distinguir, a grandes rasgos, tres áreas geolectales. La zona del seseo, señalan Narbona et al. (1998: 131), se extiende por el oeste de Huelva, el norte de Sevilla, incluyendo la capital, por casi toda la campiña cordobesa, por algunos pueblos del centro de la provincia de Jaén, áreas al norte de la provincia de Málaga, al oeste de Granada y en el sudeste de Almería. Estos autores explican que, si bien el seseo ocupa zonas más hacia el centro de Andalucía, el ceceo se ubica preferentemente por áreas costeras y se localiza en las provincias de Huelva (aproximadamente una tercera parte del territorio), Cádiz (en su totalidad), zonas meridionales de Sevilla y Málaga y localidades de la costa de Granada y Almería. En tercer lugar, el área de distinción se ha ubicado por el "norte de las provincias de Huelva, Sevilla y Córdoba, la mayor parte de las provincias de Jaén y Almería, y mitad oriental de Granada" (Jiménez 1999: 32).

Ahora bien, ese mosaico dialectal se complica si adoptamos una perspectiva sociolingüística en la que se muestre el polimorfismo interno existente en las variedades locales, especialmente en las zonas urbanas. A este respecto, traemos aquí los resultados más relevantes recopilados a partir de las investigaciones realizadas en las ciudades de Córdoba, Granada, Jerez, Málaga y la propia Sevilla.

Los datos con los que contamos hasta el momento de la capital cordobesa muestran una tendencia claramente seseante (Uruburu 1996: 248-250), con porcentajes elevados en el sociolecto alto $(89,70 \%)$, aunque son aún superiores en el nivel medio $(95,28 \%)$ y en el bajo $(98,77 \%)$. Las soluciones

\footnotetext{
2 Proyecto I+D Excelencia, convocatoria de 2015, financiado por el Ministerio de Economía y Competitividad de España (referencia FFI2015-68171-C5-3-P, MINECO/FEDER/ UE).
} 
etimológicas aparecen con mayor frecuencia en los grupos más instruidos, especialmente entre los informantes de menor edad, aunque su presencia es claramente inferior a la del seseo.

La realización de /s/ y / $/$ / en la ciudad de Granada (Salvador 1980; Moya Corral y García Wiedemann 1995; Moya Corral y Sosiński 2015) refleja la convivencia de soluciones seseantes, ceceantes y distinguidoras, con porcentajes que han ido variando a lo largo de las últimas décadas. Los datos más recientes (Moya Corral y Sosiński 2015: 51 y ss.) han puesto de manifiesto el progresivo afianzamiento de la variante distinguidora, que se ha convertido en la pauta preferida con unos índices de uso del 79,4\%, y a una considerable distancia de la segunda opción más empleada, el seseo, con una representación del 13,1\%. La pronunciación etimológica está especialmente liderada por los hablantes del sociolecto alto $(92,8 \%)$, y también muestra una situación aventajada entre los habitantes más jóvenes y entre las mujeres.

El seseo y el ceceo fueron los rasgos de pronunciación más generalizados en Jerez durante la década de los ochenta, con un índice de aceptación ${ }^{3}$ del 0.91 (Carbonero et al. 1992: 23-25). Tanto el sociolecto bajo como la primera y la tercera generación presentaban los valores más significativos de la solución ceceante, mientras que las capas más instruidas se decantaban claramente por el seseo. Por su parte, la distinción s/ $\theta$ obtuvo una tímida representación, especialmente en los niveles culto e intermedio y en la primera generación, aunque siempre con índices de aceptación que no alcanzaban el 0.2. Los datos más recientes obtenidos a partir de muestras de habla tomadas en los primeros años del siglo XXI (García-Amaya 2008: 65 y ss.) ponen de manifiesto el abandono del ceceo como opción mayoritaria en la ciudad jerezana, para dar cabida a un nuevo patrón lingüístico que promueve la adhesión al modelo de prestigio nacional con mantenimiento de las soluciones etimológicas. Este cambio se percibe especialmente entre los hablantes con mayor nivel educativo y entre las mujeres.

Para la ciudad de Málaga, Villena (1997: 98) ha demostrado la convivencia de realizaciones fonéticas convergentes hacia el estándar nacional con soluciones divergentes que tienden al refuerzo de las variantes de pronunciación regionales. Estos dos patrones están influidos por los rasgos sociales de los hablantes. En concreto, el mantenimiento de la distinción etimológica está liderado por el nivel educativo alto, las generaciones

\footnotetext{
Tanto para el habla de Jerez (Carbonero et al. 1992) como de Sevilla (Lamíquiz y Carbonero 1987; y Carbonero 2003b [2000]), este dato se obtuvo a partir de la división del número de hablantes que emplearon este fenómeno entre el total de informantes que conformaron la muestra.
} 
más jóvenes y las mujeres. Por otra parte, las soluciones vernáculas (bien seseantes o bien ceceantes) presentan un mayor asentamiento en informantes de más edad, de menor nivel educativo y en los hombres. Estos datos están en consonancia con los registrados de forma específica en el barrio de Capuchinos de la ciudad malagueña (Ávila 1994).

En lo que respecta a Sevilla, la capital andaluza ha sido tradicionalmente identificada como núcleo seseante (Navarro Tomás et al. 1933: 239; Alvar et al. $\left.1973^{4}\right)$. No obstante, las investigaciones sociolingüísticas han venido a demostrar que, lejos de darse una solución única, en esta ciudad conviven los rasgos de distinción, seseo y ceceo con heterogénea representación según la caracterización social de los hablantes (Lamíquiz y Carbonero 1987: 36-39). Concretamente, los datos con los que contamos hasta el momento corroboran el fuerte asentamiento del seseo, con índices de aceptación cercanos al 90\% de media en los tres niveles socioculturales. Por su parte, la solución ceceante fue poco representativa y solo se registraron casos en el sociolecto bajo. A su vez, en la norma culta el seseo compite con la distinción, con claro predominio del primero (Carbonero 2003b [2000]: 116-117).

Según se ha podido comprobar, en las capas más cultivadas de las zonas analizadas conviven rasgos de pronunciación vernáculos, principalmente el seseo, con la distinción $\mathrm{s} / \theta$, solución esta última más característica del modelo septentrional. Las ciudades de Córdoba y Sevilla se decantan por el seseo, mientras que en Granada, Málaga y parece ser que también en Jerez, se aprecia un mayor asentamiento de la distinción. En este sentido, Carbonero (2003b [2000]: 117) ha dicho que existe "al menos en las ciudades andaluzas más occidentales una tendencia al alza de la solución distinguidora". No obstante, Villena (2001: 129) puntualiza que este proceso de mímesis de realizaciones cercanas al estándar se da con mayor celeridad en las áreas andaluzas orientales y centrales que en las más occidentales, dado el prestigio del que goza entre estas últimas el seseo de Sevilla, que se proyecta sobre las zonas colindantes. De hecho, este rasgo ha sido interpretado como una marca de prestigio regional a la que se acogen, por ejemplo, las mujeres en la ciudad de Málaga (Villena 1997: 100).

Si conectamos los datos de la distribución lingüística con los rasgos sociales de los hablantes se aprecia que las capas más instruidas, las generaciones más jóvenes y, en ocasiones, las mujeres, se sitúan a la cabeza del cambio hacia una pauta de pronunciación convergente con el modelo norteño en lo que respecta al mantenimiento de la distinción etimológica 
s/ $\theta$. Asimismo, en las zonas exploradas se ha podido documentar el mayor apego a modelos de pronunciación vernáculos (seseo o ceceo) entre las capas sociales menos instruidas. Los datos recientes del sociolecto bajo de la ciudad de Sevilla se suman a esta tendencia (Santana 2016), con preferencia de la solución seseante.

\section{CUESTIONES METODOLÓGICAS}

Siguiendo los criterios de PRESEEA (Moreno Fernández 1996 y 2005), los materiales utilizados para el análisis están compuestos por la transcripción de veinticuatro grabaciones de entrevistas semi-dirigidas realizadas entre los años 2009 y 2015 a informantes sevillanos (que hayan nacido en la ciudad o que hayan vivido en ella la mayor parte de su vida, con plena integración en las costumbres y en la cotidianidad de la urbe en este segundo caso). En ellas, un investigador encauza la conversación hacia una serie de módulos temáticos: el barrio, la familia, viajes..., con los que se genera una situación de conversación semi-formal que permite obtener un discurso oral no elaborado. Los informantes fueron clasificados atendiendo a tres parámetros sociales que se codificaron en el siguiente orden: sexo, edad y nivel sociocultural. En lo que respecta a la variable sexo, se hizo el mismo número de encuestas a hombres y a mujeres (codificación H / M). Para la edad se manejaron tres bloques: primera generación hasta treinta y cuatro años, segunda generación desde treinta y cinco hasta cincuenta y cuatro y tercera generación de cincuenta y cinco en adelante, que se codifican como 1, 2 y 3 respectivamente. En tercer lugar, todos los encuestados pertenecen al nivel educativo alto (codificado como 3), es decir, tienen estudios universitarios. Finalmente, las encuestas están numeradas, en nuestro caso desde 049 hasta 072. En esta ocasión omitimos el dato relativo a la ciudad (SEVI), pues aquí no es relevante. De este modo, por ejemplo, la grabación H13-049 pertenece a un hombre de la primera generación del nivel educativo alto con entrevista número 049.

Para llevar a cabo esta investigación se han tomado las siguientes decisiones:

a) Los fenómenos de distinción, seseo y ceceo implican el estudio conjunto de dos variables fonológicas: $/ \mathrm{s} / \mathrm{y} / \theta /$. De este modo, para el análisis cuantitativo sería preciso considerar las variantes de ambos segmentos de forma separada. No obstante, en una primera fase del 
análisis comprobamos que en nuestro corpus todos los casos de /s/ obtuvieron la solución [s]. Por tanto, no se documentaron casos de ceceo en la norma culta sevillana. Hay coincidencia plena con los datos obtenidos a partir de grabaciones de los años 1972 y $1973^{5}$ (Carbonero 2003a [1985]: 25; Lamíquiz y Carbonero 1987: 36), por lo que se descarta que sea un fenómeno presente o en avance en las clases más instruidas en la ciudad de Sevilla. Por esta razón no hemos cuantificado la variable $/ \mathrm{s} /$. De este modo, las cifras de mantenimiento se refieren a $/ \theta /$ como $[\theta]$, pues fue el único segmento donde se dio la alternancia de más de una solución. Sin embargo, cabe decir que las realizaciones de $/ \theta /$ como $[\theta]$ pueden considerarse a su vez casos de distinción de $s / \theta$ pues, como se ha dicho, esos informantes también emplearon $/ \mathrm{s} /$ como [s].

b) Para cuantificar los fenómenos de seseo y de ceceo sería lícito pensar que los casos de /s/ como [s] en hablantes plenamente seseantes son también ejemplos de esta realización fonética y, consecuentemente, que los de $/ \theta /$ como $[\theta]$ serían ocurrencias de ceceo en los hablantes solo ceceantes. Pero, ¿cómo clasificaríamos, por ejemplo, los usos de /s/ como [s] en aquellos informantes que combinan en su pronunciación fragmentos seseantes con fragmentos de distinción etimológica? En aras de evitar confusiones, siguiendo a Moya Corral y García Wiedemann (1995: 146), para nuestra investigación solo consideraremos casos de seseo las realizaciones de / $\theta /$ como [s]. Así evitamos interpretaciones subjetivas sobre posibles casos de seseo o mantenimiento cuando no contamos con criterios sólidos para decantarnos por una u otra opción.

c) En lo que respecta a la identificación de las variantes, hemos de decir que no siempre fue sencillo reconocer la realización fonética que empleó el hablante. Esto se debe fundamentalmente a que, aunque en principio se perciben con bastante claridad las soluciones de seseo, ceceo y distinción, en realidad esto se materializa "en toda una gama de matices intermedios con distintos modos de articulación" (Carbonero et al. 1992: 23). Por esta razón, a sabiendas de que puede haber cierto margen de error para los casos dudosos (que son pocos), hemos considerado como [s] aquellos alófonos de / $\theta$ / que se acercan 
más hacia la realización siseante y como $[\theta]$ a los que se acercan más claramente a la ciceante. Los ejemplos en los que nos resultó imposible decantarnos por una u otra solución fueron eliminados del estudio ${ }^{6}$.

d) En cuanto a la cantidad de material empleado, seleccionamos los veinte primeros minutos de cada grabación. A este respecto cabe señalar que a menudo se descarta el comienzo del audio para el análisis lingüístico, pues se considera que en esa parte de la entrevista el discurso del hablante es menos natural al sentirse todavía intimidado por el hecho de que está siendo grabado. Esa sensación, sin embargo, se suele ir perdiendo conforme va avanzando la encuesta, especialmente si existe una relación de cercanía entre los interlocutores y si se consigue abordar temas con los que el informante se encuentra cómodo. Ese grado de conciencia sobre la propia producción lingüística, aspecto que para otras investigaciones podría ser un inconveniente, en este caso fue un factor de interés. A nuestro modo de ver, es posible que en esos primeros momentos el hablante se esfuerce por emplear una pronunciación más esmerada, más cercana a modelos ideales de prestigio, decisión que puede ir abandonando progresivamente conforme avance la grabación y se vaya sintiendo más relajado y confiado. Esta característica, que hemos observado en algunos encuestados (H23-056), nos aportaría indicios para la explicación del nivel de vacilación en un mismo hablante. Es decir, nos permitiría aventurar que en situaciones de formalidad se emplean variantes de pronunciación distintas a las que se seleccionan en contextos más informales.

\section{ANÁLISIS DE LA MUESTRA}

Siguiendo la línea de lo que se ha expuesto anteriormente, las realizaciones de $/ \theta /$ en inicio de sílaba no se extienden de forma homogénea entre los

\footnotetext{
6 La falta de claridad en estos casos se debió a la rapidez en la enunciación o al solapamiento del segmento que nos interesaba con un ruido de fondo o con la intervención del entrevistador.
} 
hablantes sevillanos. Veremos cómo la alternancia de soluciones se distribuye de manera dispar entre los encuestados (con combinación de más de una solución incluso en un mismo hablante). Para dar cuenta de esta diversidad consideraremos el índice de representación de seseo y distinción, de forma global, en esta comunidad de habla y en este sociolecto. Esto es, en qué proporción se reparten tales fenómenos entre los hablantes cultos sevillanos. Pero además deberemos dar cuenta del polimorfismo existente basándonos en el grado de distribución entre los informantes (qué porcentaje prefiere una solución u otra) y en el nivel de uniformidad que dichas variantes tienen según cada individuo (qué porcentaje emplea una única solución o vacila entre ambas, así como qué índices de frecuencia revela dicha vacilación). De este modo podremos combinar un panorama general de la presencia de estos rasgos de pronunciación en la norma culta sevillana con una visión más pormenorizada que ponga de manifiesto la heterogeneidad de su distribución. Por último nos planteamos abordar dicho polimorfismo desde un punto de vista sociolingüístico, de forma que podamos apreciar si los rasgos sociales de edad y sexo condicionan los procesos de variación que se detecten.

\subsection{Distribución DE LAS REALIZACIONES DE / $\theta$ / EN INICIO DE SílaBA}

Tabla 1. Distribución global de seseo y mantenimiento

\begin{tabular}{|l|c|c|}
\cline { 2 - 3 } \multicolumn{1}{c|}{} & N & $\%$ \\
\hline Seseo & 829 & 25,54 \\
\hline Mantenimiento & 2417 & 74,46 \\
\hline TOTAL & \multicolumn{2}{|c|}{3246} \\
\hline
\end{tabular}


Gráfico I. Distribución global de seseo y mantenimiento

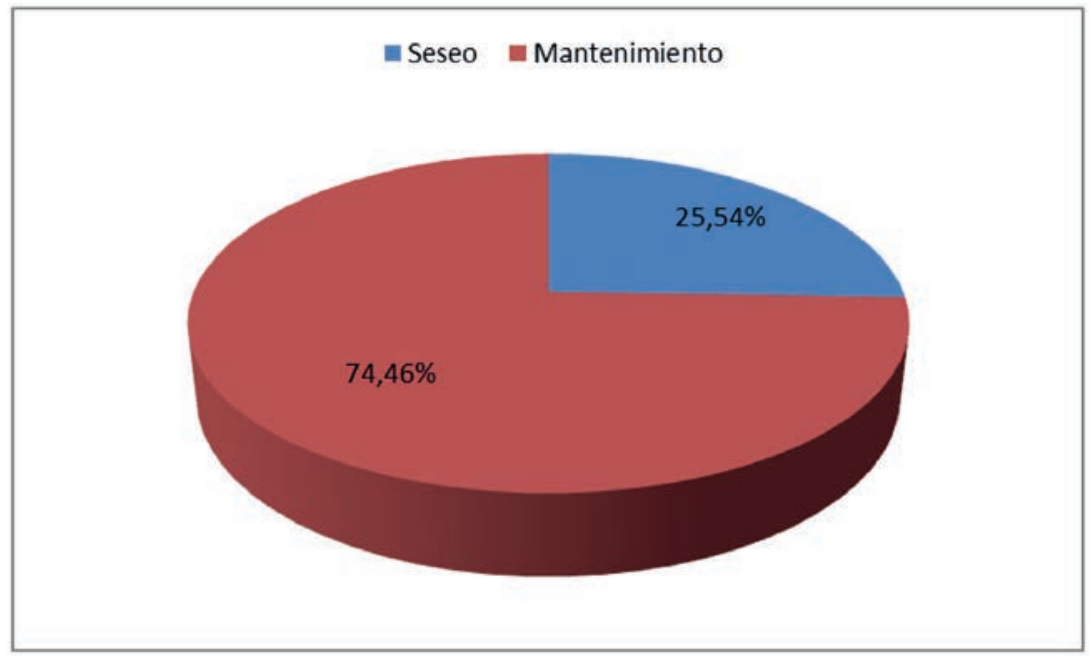

Como se puede apreciar, es clara la preferencia por el mantenimiento de la pronunciación etimológica en el sociolecto alto sevillano. Los porcentajes muestran un claro asentamiento de esta solución. Si hacemos una comparación con los resultados de décadas precedentes (Carbonero 2003a [1985]: 25; Lamíquiz y Carbonero 1987: 42) se aprecia un cambio significativo: mientras que en los años setenta el seseo obtuvo unos índices de aceptación mayoritarios ( $70 \%$ y $74 \%$, en uno y otro estudio), en las encuestas más recientes apenas ha rebasado el $25 \%$. En términos generales puede afirmarse que se está produciendo un cambio de la pauta de pronunciación de $/ \theta /$ en inicio de sílaba en la norma culta de la ciudad hispalense: frente al modelo de prestigio del andaluz, con el seseo como uno de sus patrones de pronunciación más significativos en su vertiente occidental, el cual históricamente ha tenido su eje central en la ciudad hispalense, en la actualidad se documenta empíricamente el afianzamiento del modelo de prestigio norteño entre los hablantes con mayor grado de instrucción, con la distinción etimológica como opción más representativa.

Ahora bien, según hemos venido apuntando, es previsible que los datos obtenidos tengan una distribución heterogénea entre los informantes de la muestra. Veamos en primer lugar qué número de encuestados ha utilizado de forma preferente una u otra solución, lo que denominaremos nivel de integración. Esto es, en qué medida un determinado rasgo constituye un patrón preferido que, en consecuencia, está plenamente integrado en los hábitos lingüísticos de los hablantes. 
Tabla 2. Nivel de integración de seseo y mantenimiento

\begin{tabular}{|l|c|c|}
\cline { 2 - 3 } \multicolumn{1}{c|}{} & $\mathbf{N}$ & $\%$ \\
\hline Seseo & 6 & 25,00 \\
\hline Mantenimiento & 18 & 75,00 \\
\hline TOTAL & \multicolumn{2}{|c|}{24} \\
\hline
\end{tabular}

Gráfico II. Nivel de integración de seseo y mantenimiento

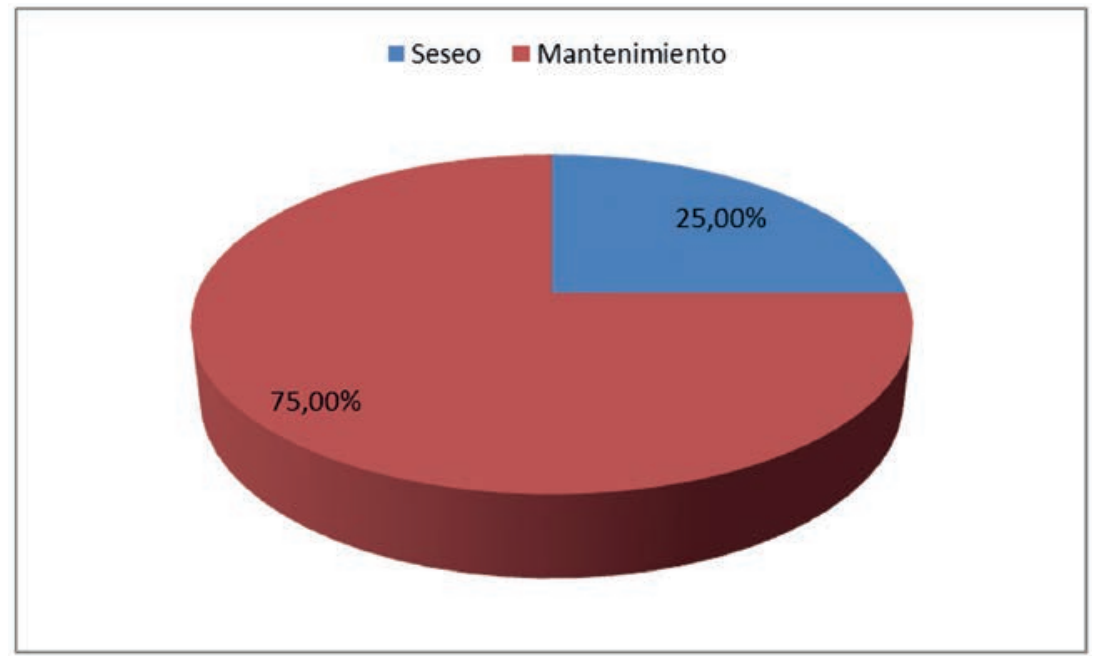

Según se observa, un elevado porcentaje de los encuestados utilizó como opción única o mayoritaria la distinción $(75 \%)$, con lo que concluimos que se trata de la variante más integrada en el sociolecto alto sevillano. Estas cifras nos llevan en la misma dirección que lo que mostraron los valores generales: la tendencia en la norma culta sevillana en la actualidad es seguir el modelo estándar nacional, con el mantenimiento de s/ $\theta$, frente al progresivo descenso que ha experimentado la solución seseante, que tradicionalmente se ha considerado como pauta de prestigio en esta urbe.

Profundizando más en la heterogénea repartición de seseo y distinción, prestaremos atención seguidamente al grado de uniformidad de estas variantes según cada informante. Es decir, en qué medida los encuestados manejan en su producción lingüística solo uno de los rasgos de pronunciación o combinan ambos, lo que denominamos nivel de seguridad. 
Tabla 3. Nivel de seguridad de seseo y mantenimiento

\begin{tabular}{lcccccc}
\hline & $\begin{array}{c}\mathbf{N}^{0} \text { informantes solo } \\
\text { o mayoritariamente } \\
\text { seseantes }\end{array}$ & $\begin{array}{c}\mathbf{N}^{\mathbf{0}} \text { informantes solo } \\
\text { o mayoritariamente } \\
\text { distinguidores }\end{array}$ & SUBTOTAL \\
\hline Una solución & $\mathrm{N}$ & $\%$ & $\mathrm{~N}$ & $\%$ & $\mathrm{~N}$ & $\%$ \\
Dos soluciones & 1 & 20,00 & 4 & 80,00 & 5 & 20,83 \\
TOTAL & 5 & 20,83 & 14 & 73,68 & 19 & 79,17 \\
\hline
\end{tabular}

Gráfico III. Nivel de seguridad de seseo y mantenimiento

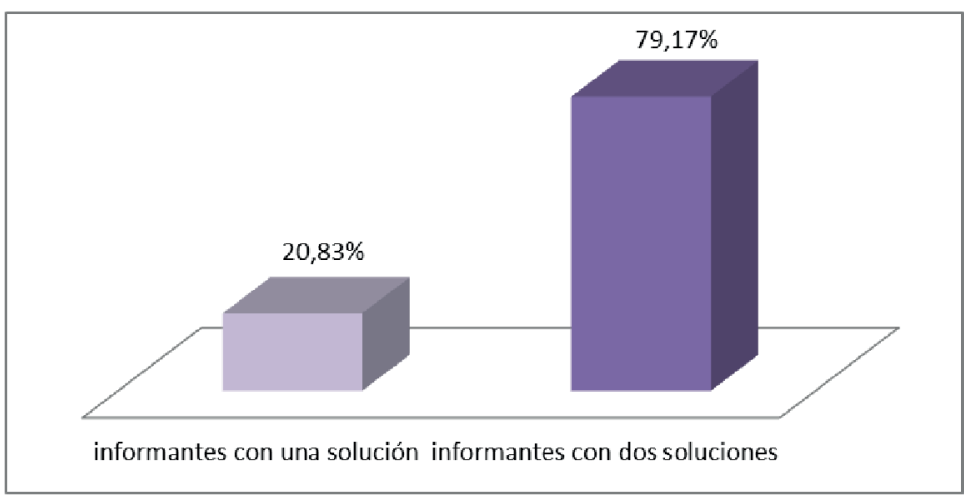

Los datos obtenidos de la muestra nos permiten hacer la diferenciación entre dos bloques de hablantes: un grupo minoritario empleó solo una de las realizaciones $(5 / 24,20,83 \%)$, mientras que la mayor parte de los encuestados combinó ambas opciones $(19 / 24,79,17 \%)$, con diferentes porcentajes de representación de cada variante. A este respecto hay que decir que el factor de inseguridad en la realización $/ \theta /$ es el predominante en esta comunidad de habla, pues la mayoría de los informantes manejó en sus intervenciones más de una realización.

Concretando algo más, el mantenimiento registró un índice de seguridad más representativo, pues fue el rasgo empleado en cuatro de los cinco hablantes que solo utilizaron una de las variantes $(80 \%)$.

Los resultados obtenidos en algunos encuestados que manejaron ambas soluciones contribuyen también a reforzar la tesis del afianzamiento de la distinción etimológica. De hecho, fue la opción preferida por un $73,68 \%$ (14/19) de aquellos hablantes que emplearon las dos variantes de pronunciación en sus intervenciones. Además, los porcentajes de uso en estos informantes fueron siempre superiores al $75 \%$. 
Un nuevo dato que revela la importancia de la solución etimológica es que en cinco ocasiones la vacilación en hablantes predominantemente distinguidores se redujo a solo un caso de seseo (M13-063 y 064; M23067) o dos (H23-054, H33-060). No descartamos que en esos individuos tales realizaciones seseantes hayan podido ser excepcionales o incluso una confusión propia de la dicción espontánea, sin que pueda decirse que sea un rasgo que caracterice habitualmente su pronunciación.

Tratando de buscar una explicación lingüística para estos ejemplos de seseo en hablantes predominantemente distinguidores, podríamos pensar que el contexto fónico en el que se sitúa $/ \theta /$ pueda haber influido. Nos referimos concretamente a la variable entorno que manejan Moya Corral y García Wiedemann (1995: 60). Estos autores diferencian entre entorno simple (cepillo) y doble (sanción / precisar). Hemos incluido también en este último grupo casos del tipo residencial en los que los fonemas $/ \mathrm{s} / \mathrm{y} / \theta /$ están muy próximos, pero no se emplean en sílabas consecutivas, sino con una sílaba intercalada. Teniendo en cuenta este parámetro, las realizaciones seseantes en estos hablantes aparecieron solo en dos ocasiones en entorno doble: esa zona (M13-063), una cierta sensibilidad (H33-060), mientras que el resto de las ocurrencias se dieron en entornos simples: una ciudad bastante (M13-064), tú dices y yo (M23-067), estábamos haciendo francés, trabajo de oficina (H23-054), podia hacer una (H33-060). Hemos podido comprobar que en todos los casos las realizaciones en entorno doble que emplearon estos hablantes, a excepción de las señaladas arriba, fueron pronunciadas distinguiendo $s / \theta$. De aquí se deduce que este factor no influye en la realización seseante. Es más, la mayoría de los vocablos donde se usó el seseo fueron utilizados en otras ocasiones por el mismo informante, pero manteniendo el alófono [ $\theta]$. Fue el caso de ciudad, dices, hacer y cierta, con repeticiones totales, y haciendo, con repetición parcial mediante algunas variantes morfológicas como hace o hacía.

Por su parte, el seseo solo fue utilizado de forma exclusiva por un informante (H13-049), lo que supone un porcentaje bajo tanto en relación con el grupo de encuestados que solo hicieron uso de una de las variantes $(20 \%, 1 / 5)$ como en relación con el conjunto de la muestra $(4,17 \%, 1 / 24)$.

Es reseñable que hubo un encuestado fundamentalmente seseante (H13052) que solo optó por la solución etimológica en una ocasión. Cabría pensar que el mantenimiento de esta forma podría estar influida por el hecho de que es un nombre propio (Sagrado Corazón), aunque esta hipótesis pierde solidez desde el momento en que otros casos similares empleados por este mismo hablante tuvieron realización seseante (Avenida San Francisco Javier, Nervión Plaza, Reina Mercedes), uno de ellos incluso emitido inmediatamente después que el caso de distinción antes mencionado $(y$ 
después también los dos colegios que he ido / Sagrado Cora[0]ón y Porta[s] eli están también en Nervión). Nos inclinamos por pensar que esta producción esporádica representa una confusión espontánea en la pronunciación y que no forma parte de los hábitos lingüísticos de este sujeto.

Por otro lado, el grado de asentamiento del seseo entre los cinco encuestados que vacilaron y que prefirieron este rasgo fue significativo en solo tres casos (H13-052, H23-053 y M33-069), con un porcentaje superior al 75\%. En los dos informantes restantes (H23-056 y M33-071), el grado de combinación de seseo y distinción estuvo mucho más reñido, con índices de uso muy próximos entre las dos variantes. De hecho, en H23-056 el seseo solo aventajó a la distinción en dos ejemplos $(50,55 \%)$.

Por tanto, a la luz de los datos relativos al índice de seguridad podemos afirmar que la pronunciación seseante va perdiendo vitalidad en el sociolecto alto sevillano, bien porque fue utilizada en exclusiva por solo un informante (a lo sumo dos si contemplamos la situación excepcional de H13-052 que hemos descrito arriba), bien porque también pocos encuestados la emplearon como alternativa mayoritaria y a veces con índices poco destacados.

A continuación vamos a centrarnos en aquellos informantes que registraron ambas soluciones (seseante y distinguidora), con más de dos ejemplos en la opción minoritaria (los casos de una o dos ocurrencias ya los hemos analizado arriba), para comprobar si hay factores lingüísticos que pudieron influir en la utilización de uno u otro recurso. Concretamente nos fijaremos en tres parámetros: el entorno (simple o doble), la lexía (si hay coincidencia o no de pronunciación en algunas palabras que se repiten de forma idéntica o que presentan algunas variaciones morfológicas, como por ejemplo: decir, decía, dice) y sustantivo común o propio. Cuando sea relevante distribuiremos la exposición en dos grupos: posible justificación de soluciones seseantes minoritarias en hablantes mayoritariamente distinguidores y de soluciones etimológicas en hablantes mayoritariamente seseantes.

En lo que respecta al entorno en el que se da el rasgo de pronunciación, simple o doble, podría pensarse que este último favorecería los casos de seseo en informantes fundamentalmente distinguidores (las vacilaciones se producirían debido a la confusión que generaría la cercanía de ambos alófonos); o bien cabría la interpretación de que la distinción en encuestados mayoritariamente seseantes sería producto de un intento de separar un fonema de otro (en este contexto el hablante es más consciente de la necesidad de diferenciar entre las unidades que a menudo neutraliza en una única solución seseante). Los resultados de la muestra revelan que este factor no influyó en la alternancia seseo-distinción en un mismo sujeto. En concreto, los casos de seseo en entornos dobles que se dieron en informantes 
mayoritariamente distinguidores fueron poco representativos en relación con el total de voces que estos encuestados utilizaron en dicho contexto fónico $(15,09 \%, 16 / 106)$. Por su parte, la frecuencia relativa de casos de distinción en entornos dobles en hablantes principalmente seseantes fue ligeramente más significativa $(18,52 \%, 10 / 54)$, pero siguió siendo un índice más bajo que el de cuarenta y cuatro casos con solución seseante.

En segundo lugar nos vamos a fijar en si aquellas voces que se emplearon de forma minoritaria con seseo o con distinción fueron repetidas en la intervención de un mismo hablante pero con otra pronunciación o si estos vocablos fueron utilizados solo con ese rasgo. Del total de ejemplos de soluciones seseantes en los hablantes principalmente distinguidores que aquí estamos considerando, un 40,29\% (56/139) fueron casos de palabras que el informante empleó siempre con esa variante. De forma similar, el $39,77 \%$ (68/171) de los casos de palabras con pronunciación distinguidora en informantes mayoritariamente seseantes fueron lexías que se emplearon exclusivamente con esa pronunciación. Aunque en ambos bloques el porcentaje fue destacado, siempre fue menor al de palabras que se repitieron con dos pronunciaciones distintas en un mismo informante $(83 / 139,59,71 \% \mathrm{y}$ $103 / 171,60,23 \%)$. Estos datos nos permiten afirmar que la utilización de uno u otro acento en aquellos hablantes que combinan ambas variantes (seseante y distinción) no depende necesariamente de la lexía, pues la mayoría de las palabras empleadas con el alófono minoritario también fueron utilizadas por el mismo informante con la otra pronunciación en alternancia.

Un tercer aspecto que hemos creído que podría servir para explicar la elección de la variante seseante o distinguidora en un mismo hablante es el hecho de que se tratara de un nombre propio. De forma general, la tendencia mayoritaria fue emplear la pronunciación etimológica en esos casos, concretamente en un porcentaje del 81,25\% (26/32). Es más, esa fue la opción preferida incluso en aquellos encuestados mayoritariamente seseantes. Solo en una ocasión, en la intervención de un hombre de la segunda generación (H23-053), la variante no distinguidora fue la más empleada. Es palpable que estos sustantivos, con referentes individualizados, se usan preferentemente con su pronunciación originaria, como era previsible. Se trata, por tanto, de un factor que puede haber condicionado la elección de la distinción etimológica en aquellos encuestados que alternaron las dos variantes, independientemente de cuál de ellas fuera la opción mayoritaria. 


\section{DistribuCiÓN SOCIOLINGÜÍSTICA}

DE LAS REALIZACIONES DE / $\theta$ / EN INICIO DE SílABA

En último lugar vamos a considerar la relación entre la alternancia de seseo - realización etimológica de $/ \theta /$ y los rasgos sociales de los informantes: edad y sexo.

Tabla 4. Seseo y mantenimiento según edad

\begin{tabular}{lccccc}
\hline & \multicolumn{2}{c}{ Seseo } & \multicolumn{2}{c}{ Mantenimiento } & \multirow{2}{*}{ TOTAL } \\
\cline { 1 - 4 } & $\mathrm{N}$ & $\%$ & $\mathrm{~N}$ & $\%$ & \\
$\mathbf{1}^{\mathbf{a}}$ gen. & 266 & 25,77 & 766 & 74,22 & 1032 \\
$\mathbf{2}^{\mathbf{a}}$ gen. & 249 & 22,13 & 876 & 77,87 & 1125 \\
$\mathbf{3}^{\text {a }}$ gen. & 314 & 28,83 & 775 & 77,17 & 1089 \\
\hline
\end{tabular}

Gráfico IV. Seseo y mantenimiento según edad



Tabla 5. Nivel de integración de seseo y mantenimiento según edad

\begin{tabular}{|c|c|c|c|c|c|}
\hline & \multicolumn{2}{|c|}{$\begin{array}{l}\text { Informantes que } \\
\text { prefieren seseo }\end{array}$} & \multicolumn{2}{|c|}{$\begin{array}{c}\text { Informantes que prefieren } \\
\text { mantenimiento }\end{array}$} & \multirow[t]{2}{*}{ TOTAL } \\
\hline & $\mathrm{N}$ & $\%$ & $\mathrm{~N}$ & $\%$ & \\
\hline $1^{\text {a }}$ gen. & 2 & 25 & 6 & 75 & 8 \\
\hline $2^{\mathrm{a}}$ gen. & 2 & 25 & 6 & 75 & 8 \\
\hline $3^{\text {a }}$ gen. & 2 & 25 & 6 & 75 & 8 \\
\hline
\end{tabular}


Gráfico V. Nivel de integración de seseo y mantenimiento según edad



La preferencia por la pronunciación etimológica es un rasgo generalizado que, además, no revela diferencias significativas según los grupos etarios, como se comprueba en las cifras obtenidas. En todas las generaciones se prefiere el mantenimiento de $/ \theta /$, además con porcentajes superiores al $70 \%$. El nivel de integración, por su parte, está en consonancia con ese dato, pues en todos los grupos etarios se observó el uso mayoritario o exclusivo de la variante mantenimiento. De aquí se deduce que se trata de un fenómeno ya asentado, en tanto que se da en los hablantes de mayor edad y, además, esta tendencia tiene visos de perdurar en el tiempo, pues sigue siendo la pronunciación preferida por los encuestados más jóvenes.

El grado de vacilación entre una y otra variante en los informantes que prefirieron la distinción, sin embargo, nos permite atisbar algunas diferencias entre unas generaciones y otras, según se muestra a continuación.

Tabla 6. Nivel de seguridad de seseo y mantenimiento según edad

\begin{tabular}{|c|c|c|c|}
\hline \multicolumn{4}{|c|}{ Tabla 6.1. Informantes con una solución } \\
\hline \multicolumn{2}{|c|}{ Solo seseo } & \multicolumn{2}{|c|}{ Solo mantenimiento } \\
\hline Informante & $\mathbf{N}$ & Informante & $\mathbf{N}$ \\
\hline \multirow[t]{4}{*}{ H13-049 } & 124 & H13-051 & 152 \\
\hline & & M13-062 & 152 \\
\hline & & M23-066 & 167 \\
\hline & & M23-068 & 118 \\
\hline
\end{tabular}




\begin{tabular}{lcccc}
\hline Tabla 6.2. Informantes con dos soluciones & \multicolumn{2}{c}{} \\
\hline \multicolumn{4}{c}{ Predomina seseo } \\
Informante & Neseo & \% & Mantenimiento \\
H13-052 & 125 & 99,22 & N & \% \\
H23-053 & 142 & 96,60 & 5 & 0,8 \\
H23-056 & 91 & 50,55 & 89 & 3,4 \\
M33-069 & 127 & 76,04 & 40 & 49,44 \\
M33-071 & 74 & 66,66 & 37 & 23,95 \\
& Predomina mantenimiento & & 33,33 \\
H13-050 & 8 & 4,6 & 166 & \\
M13-061 & 7 & 6,540 & 100 & 95,42 \\
M13-063 & 1 & 0,97 & 102 & 93,45 \\
M13-064 & 1 & 1,06 & 93 & 99,03 \\
H23-054 & 2 & 2,53 & 77 & 98,93 \\
H23-055 & 9 & 7,32 & 114 & 97,46 \\
M23-065 & 4 & 3 & 129 & 92,68 \\
M23-067 & 1 & 0,54 & 181 & 96,99 \\
H33-057 & 37 & 22,83 & 125 & 99,45 \\
H33-058 & 32 & 24,06 & 101 & 77,16 \\
H33-059 & 25 & 20 & 100 & 85,93 \\
H33-060 & 2 & 1,21 & 163 & 98,78 \\
M33-070 & 3 & 12,72 & 96 & 84,21 \\
M33-072 & 2,58 & 113 & 97,41 \\
\hline
\end{tabular}

Los grupos etarios de mayor edad fueron los que se mostraron más inseguros, aspecto que se deduce a partir de los siguientes datos:

1. Todos los encuestados que emplearon solo seseo o solo mantenimiento de $/ \theta /$ pertenecen a la primera y a la segunda generación (H13-049; H13-051; M13-062; M23-066; M23-068). Cabe señalar también que aquellos hablantes que vacilaron en un único ejemplo, es decir, que son, en términos generales, distinguidores o seseantes, se ubican también entre las generaciones más jóvenes (H13-052; M13-063, M13-064, M23-067).

2. Los usuarios con un mayor grado de inseguridad, es decir, con porcentajes más cercanos y equilibrados entre las dos variantes de pronunciación, pertenecen mayoritariamente a la tercera generación (H33-057, H33-058; M33-069; M33-071), aunque el caso más destacado es un hombre de la segunda (H23-056). 
Por tanto, los informantes más jóvenes muestran mayor seguridad en la elección de la distinción etimológica, mientras que se producen más dudas y vacilaciones conforme vamos avanzando en la edad.

Veamos a continuación en qué medida la variable sexo influyó en la distribución de nuestras dos variantes.

Tabla 7. Seseo y mantenimiento según sexo

\begin{tabular}{|c|c|c|c|c|c|}
\hline & \multicolumn{2}{|c|}{ Seseo } & \multicolumn{2}{|c|}{ Mantenimiento } & \multirow{2}{*}{ TOTAL } \\
\hline & $\mathrm{N}$ & $\%$ & $\mathrm{~N}$ & $\%$ & \\
\hline Hombres & 597 & 35,33 & 1093 & 64,67 & 1690 \\
\hline Mujeres & 232 & 14,91 & 1324 & 85,09 & 1556 \\
\hline
\end{tabular}

Gráfico VI. Seseo y mantenimiento según sexo

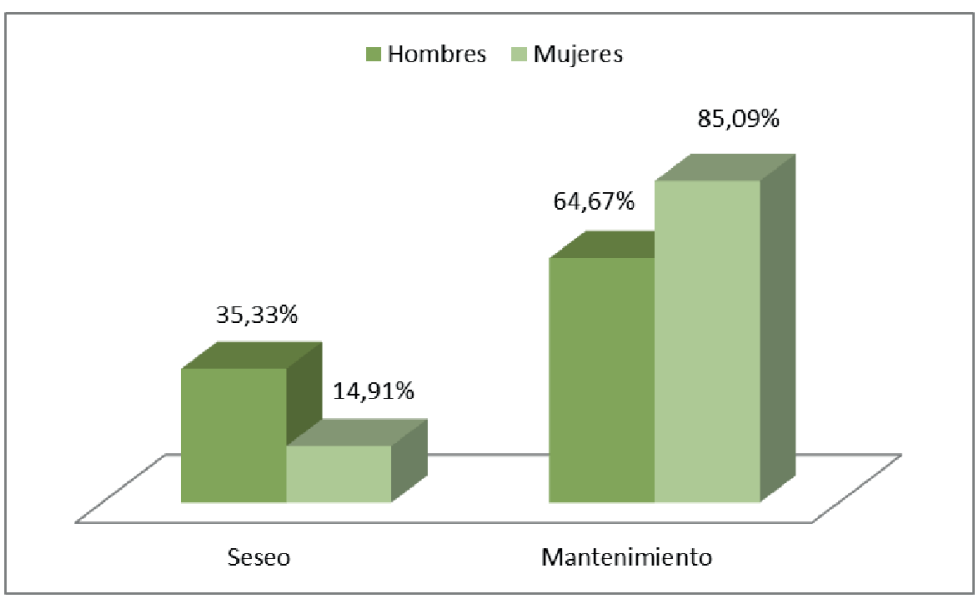

Tabla 8. Nivel de integración de seseo y mantenimiento según sexo

\begin{tabular}{lccccc}
\hline & \multicolumn{2}{c}{$\begin{array}{c}\text { Informantes que } \\
\text { prefieren seseo }\end{array}$} & \multicolumn{2}{c}{$\begin{array}{c}\text { Informantes que } \\
\text { prefieren mantenimiento }\end{array}$} & \multirow{2}{*}{ TOTAL } \\
\cline { 2 - 4 } Hombres & $\mathbf{N}$ & $\mathbf{\%}$ & $\mathbf{N}$ & $\mathbf{\%}$ & \\
Mujeres & 4 & 33,33 & 8 & 66,67 & 12 \\
\cline { 1 - 3 } & 2 & 20,00 & 10 & 80,00 & 12 \\
\hline
\end{tabular}


Gráfico VII. Nivel de integración de seseo y mantenimiento según sexo

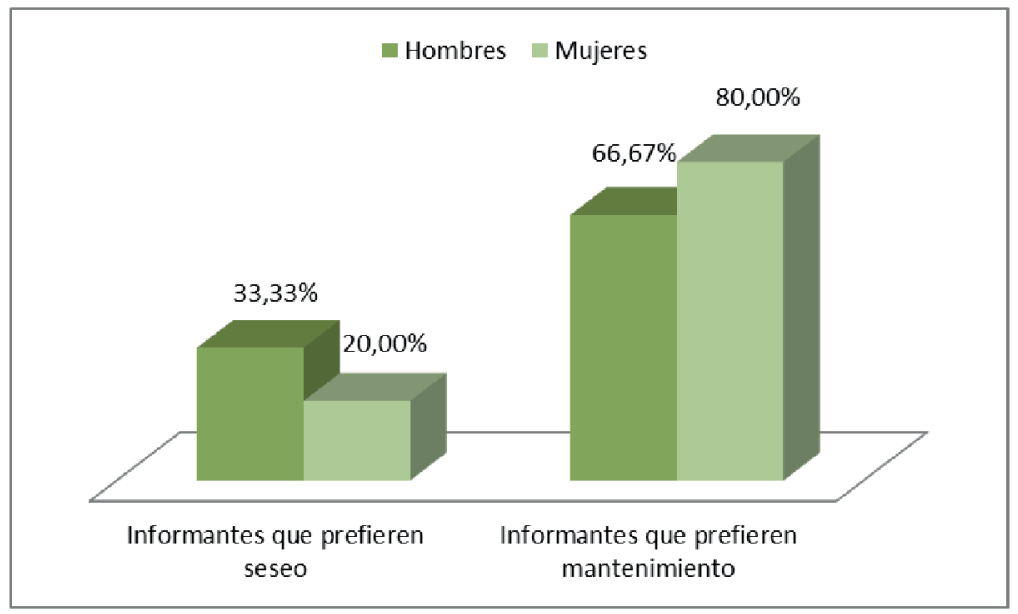

Según se observa, la tendencia al mantenimiento de $/ \theta /$ es común a ambos sexos. No obstante, dicha preferencia está más consolidada en las mujeres que en los hombres. Es decir, son las féminas las que muestran un mayor apego a la pronunciación etimológica, rasgo conservador y de convergencia hacia la modalidad septentrional. Podríamos decir que se cumple una pauta repetida en otros estudios sociolingüísticos: las mujeres se acercan más a las formas estándares, especialmente cuando tienen una consideración más prestigiosa que otras variantes en alternancia (López Morales 2004 [1989]: 127-130). El grado de integración, que se muestra en la tabla 8 y en el gráfico VII, también indica que el porcentaje de mujeres que prefirieron el mantenimiento fue superior al de hombres.

Otro aspecto interesante es observar el nivel de seguridad en relación con la variable sexo. Para ello volvemos sobre los datos expuestos en la tabla 6 , donde a la preferencia de las mujeres por la variante etimológica, según se ha dicho, se suma el hecho de que son ellas también las que muestran un menor índice de vacilación. Señalamos varios datos que nos han permitido llegar a esta conclusión:

1. De los cuatro informantes que emplearon de forma exclusiva la pronunciación etimológica, un 75\% fueron féminas (M13-062, M23066; M23-068). Por su parte, el único sujeto plenamente seseante fue un hombre (H13-049).

2. Cuando se produjo vacilación entre las dos variantes, aunque no puede decirse que haya una diferencia determinante por sexos, sí se 
aprecia que la media de porcentajes de preferencia de las mujeres por el mantenimiento $(95,64 \%)$ es superior a la de los hombres $(88,2 \%)$, aunque ambos índices son bastante elevados. Es decir, las féminas muestran más seguridad al decantarse por el mantenimiento de la pronunciación etimológica y, de forma paralela, la media de seseo es menor en ellas $(3,92 \%)$ y sensiblemente más destacada en ellos $(11,79 \%)$. Reforzando estos datos, los índices de preferencia por la opción seseante en los casos de vacilación obtuvo más fuerza en los hombres $(82,12 \%)$, seguido de cerca por las mujeres $(71,35 \%)$.

Finalmente, si cruzamos los datos de edad y de sexo se pueden obtener algunos resultados relevantes.

Tabla 9. Seseo y mantenimiento según edad y sexo ${ }^{7}$

\begin{tabular}{|c|c|c|c|c|c|c|c|c|c|}
\hline \multicolumn{5}{|c|}{ Hombres } & \multicolumn{5}{|c|}{ Mujeres } \\
\hline Informante & Seseo & Media & Manten & Media & Informante & Seseo & Media & Manten. & Media \\
\hline H13-049 & 100 & & & & M13-061 & 6,54 & & 93,45 & \\
\hline H13-050 & 4,60 & \multirow{2}{*}{50,96} & 95,42 & \multirow{3}{*}{49,06} & M13-062 & & & 100,00 & \multirow{3}{*}{97,85} \\
\hline H13-051 & & & 100,00 & & M13-063 & 0,97 & & 93,03 & \\
\hline H13-052 & 99,22 & & 0,80 & & M13-064 & 1,06 & & 98,93 & \\
\hline H23-053 & 96,60 & & 3,40 & \multirow{4}{*}{60,75} & M23-065 & 3,00 & & 96,99 & \\
\hline H23-054 & 2,53 & \multirow{3}{*}{39,25} & 97,46 & & M23-066 & & \multirow{3}{*}{0,88} & 10,000 & \multirow{3}{*}{99,11} \\
\hline H23-055 & 7,32 & & 92,68 & & M23-067 & 0,54 & & 99,45 & \\
\hline H23-056 & 50,55 & & 49,44 & & M23-068 & & & 100,00 & \\
\hline H33-057 & 22,83 & & 77,16 & \multirow{4}{*}{82,97} & M33-069 & 76,04 & & 23,95 & \multirow{4}{*}{59,73} \\
\hline H33-058 & 24,06 & \multirow{2}{*}{17,03} & 75,93 & & M33-070 & 12,72 & & 84,21 & \\
\hline H33-059 & 20,00 & & 80,00 & & M33-071 & 66,66 & 39,5 & 33,33 & \\
\hline H33-060 & 1,21 & & 98,78 & & M33-072 & 2,58 & & 97,41 & \\
\hline
\end{tabular}

Las mujeres de la segunda generación fueron las que lideraron la preferencia por la solución etimológica, seguidas muy de cerca por el grupo de féminas de 
menor edad. Los porcentajes de uso son muy altos (unas medias de $99,11 \%$ y $97,85 \%$, respectivamente). En consecuencia, los niveles de vacilación fueron bajos en estos grupos socioculturales, con índices mínimos representación del seseo (una media de $0,88 \%$ y $2,14 \%$ para cada caso), los más bajos entre todos los encuestados. Recuérdese que aquí se sitúan también las tres mujeres que fueron plenamente distinguidoras (M13-062, M23-066 y M23-068). Por su parte, los hombres del grupo etario más joven fueron los abanderados del seseo, con dos informantes que se decantaron plenamente y casi al $100 \%$ por esta variante (H13-049 y H13-052). Ahora bien, tanto las mujeres de la tercera generación como los hombres de la segunda presentaron índices de seseo también relevantes, y en estos grupos se percibió mayor homogeneidad en tanto que todos los encuestados registraron casos de esta variante, y en la mitad de ellos fue la opción preferida.

\section{CONCLUSIONES}

El estudio de la realización de $/ \theta /$ en situación inicial de sílaba en el sociolecto alto de Sevilla reveló la existencia de dos variantes en alternancia: mantenimiento y seseo. Los resultados del análisis presentan la pronunciación etimológica como el rasgo más representativo, es decir, el que goza de una mayor extensión en este grupo de hablantes. Se trata también del fenómeno con un nivel de integración más alto (fue el preferido por la mayoría de los encuestados). Dicha preferencia se pone de manifiesto, además, en todos los grupos sociales, aunque se percibe un refuerzo de esta tendencia en las mujeres de edades más jóvenes. En términos generales queda patente que con la distinción s/ $\theta$ los hablantes cultos sevillanos se acercan al modelo de prestigio nacional, más próximo a las soluciones del español norteño, mientras que dejan en un segundo plano la opción característica del patrón de prestigio meridional, el seseo. Los datos de nuestros materiales confirman las palabras de Carbonero (2003a [1985]: 22): "Los niveles cultos de la sociedad y las situaciones de mayor consideración social suelen dejar de lado los particularismos dialectales y propician las realizaciones estandarizadas".

Si se comparan los resultados de esta investigación, obtenidos a partir de los materiales de PRESEEA-Sevilla recogidos entre 2009 y 2015, con los que se recopilaron en los años setenta, se percibe un cambio importante: frente a unos índices en torno al $70 \%$ del seseo hace cuatro décadas, en la actualidad ese puesto lo ocupa la variante etimológica, con un porcentaje del $75 \%$. No obstante, un dato reseñable es que el seseo reveló una pervivencia 
más destacada en los hombres de la primera generación. Con ello se intuye que la lucha entre las dos variantes se mantiene viva en los grupos etarios más jóvenes, por lo que es previsible que perdure como una característica de la pronunciación de los sectores más instruidos.

La realización de $/ \theta /$ iniciando sílaba en el sociolecto alto sevillano no presenta una distribución homogénea. Muy al contrario, está latente una constante vacilación entre la solución convergente que prefiere la pronunciación etimológica y la solución divergente que refuerza el rasgo dialectal del seseo. La combinación de las dos variantes en un mismo hablante fue la fórmula más extendida, por lo que se convierte en un rasgo característico de esta modalidad de habla local y de este sociolecto. Los mayores índices de inseguridad se detectaron en los informantes de la tercera generación, tanto hombres como mujeres, pues fue el grupo etario en el que la alternancia se dio en todos los encuestados y, además, en la mayoría de ellos con porcentajes más equilibrados ambas soluciones que en otros grupos de edad. Esto es, que de forma habitual, y no de manera esporádica, un número destacado de estos informantes alternó en su producción lingüística el seseo y el mantenimiento de $/ \theta /$.

Ante la búsqueda de una posible causa que explicara los usos minoritarios de seseo en sujetos preferentemente distinguidores o de distinción en los mayoritariamente seseantes, nos fijamos en los factores de entorno de aparición del fonema (simple o doble), en la lexía (si estaba asociado el tipo de pronunciación a una palabra específica) y en la diferencia entre nombre común y nombre propio. Este último fue el único factor que, a juzgar por nuestros datos, influyó en la elección del hablante. Concretamente, los nombres propios tendieron a mantener la pronunciación etimológica originaria, incluso en informantes que se decantaron por el seseo.

Concluimos esta investigación destacando la preferencia en el sociolecto alto sevillano de la distinción $\mathrm{s} / \theta$, principalmente en los grupos etarios de menor edad y en las mujeres. Se percibe, por tanto, el avance de un proceso de convergencia hacia el patrón de prestigio del estándar, en detrimento de la variante vernácula seseante. Queda por saber si esta tendencia se mantiene en otros niveles socioculturales, aspecto sobre el que seguiremos profundizando en ulteriores investigaciones. 


\section{REFERENCIAS BIBLIOGRÁFICAS}

Ávila, Antonio. 1994. La variación reticular e individual de s/z en el vernáculo urbano malagueño. Datos del barrio de Capuchinos. Analecta Malacitana 17/2: 343-367.

Alvar, Manuel, Antonio Llorente y Gregorio Salvador. 1973. Atlas lingüístico y etnográfico de Andalucía. Vol. VI. Madrid: Consejo Superior de Investigaciones Científicas.

CArbonero, Pedro. 1982. El habla de Sevilla. Sevilla: Ayuntamiento de Sevilla.

2003a [1985]. Norma estándar y actitud sociolingüística. Estudios de sociolingüística andaluza, pp. 21-29. Sevilla: Secretariado de Publicaciones de la Universidad de Sevilla (originariamente en 1985. Sociolingüistica Andaluza 1, pp. 141150. Publicaciones de la Universidad de Sevilla).

2003b [2000]. Norma culta y actitudes lingüísticas de los andaluces. Estudios de sociolingüistica andaluza, pp. 109-120. Sevilla: Secretariado de Publicaciones de la Universidad de Sevilla (originariamente en 2000. Actas del Congreso Internacional: El Español Culto en el Mundo Hispánico, pp. 25-32. Universidad Bolivariana, Santiago de Chile).

Carbonero, Pedro, José Luis Álvarez, Joaquín Casas e Isabel María Gutiérrez. 1992. El habla de Jerez. Jerez: Servicio de Publicaciones del Ayuntamiento de Jerez.

García-Amaya, LoRenzo. 2008. Variable norms in the production of / $\theta$ / in Jerez de la Frontera, Spain. En Jason F. Siegel, Traci C. Nagle, Amandine Lorente-Lapole y Julie Auger (eds.). Indiana University Working Papers in Linguistics. Vol. 7. Gender in Language: Classic Questions, New Contexts, pp. 49-71. Bloomington: IULC.

Jiménez, RAfael. 1999. El andaluz. Madrid: Arco Libros.

LamíQuiz, Vidal y Miguel Ángel Pineda. 1983. Sociolingüística Andaluza 2. Encuestas del habla urbana de Sevilla. Nivel culto. Sevilla: Publicaciones de la Universidad de Sevilla.

LamíQuiz, Vidal y Pedro Carbonero. 1987. Perfil sociolingüistico del sevillano culto. Sevilla: Instituto de Desarrollo Regional (Universidad de Sevilla).

López Morales, Humberto. 2004 [1989]. Sociolingüística. $3^{\text {a }}$ edición. Madrid: Gredos.

Moreno Fernández, Francisco. 1996. Metodología del 'Proyecto para el Estudio Sociolingüístico del Español de España y de América'(PRESEEA). Lingüística 8: 257-287. 2005. Corpus para el estudio del español en su variación geográfica y social. El corpus PRESEEA. Oralia 8: 123-140.

Moya Corral, Juan Antonio y Emilio José García Wiedemann. 1995. El habla de Granada y sus barrios. Granada: Universidad de Granada.

Moya Corral, JuAn Antonio y Marcin Sosiński. 2015. La inserción social del cambio. La distinción s/ $\theta$ en Granada. Análisis en tiempo aparente y en tiempo real. Lingüistica Española Actual XXXVII/1: 33-72.

Narbona, Antonio, Rafael Cano y Ramón Morillo. 1998. El español hablado en Andalucía. Barcelona: Ariel Lingüística.

Navarro Tomás, Tomás, Aurelio Macedonio Espinosa y Lorenzo Rodríguez Castellano. 1933. La frontera del andaluz. Revista de Filología Española 20: 225-277.

Salvador, Francisco. 1980. Niveles sociolingüísticos de seseo, ceceo y distinción en la ciudad de Granada. Español Actual 37-38: 25-32.

SANTANA, JuAna. 2016. La realización de/s/y/9/ iniciando sílaba en los materiales de PRESEEA Sevilla: estudio en el sociolecto bajo. Comunicación presentada en el XII Congreso Internacional de Lingüística General. Alcalá de Henares, España (23-25 mayo 2016).

Uruburu, Agustín. 1996. La lengua hablada en Córdoba. Revista Española de Lingüística Aplicada 11: 225-250. 
Villena, Juan Andrés. 1997. Convergencia y divergencia dialectal en el continuo sociolingüístico andaluz: datos del vernáculo urbano malagueño. Lingüística Española Actual XIX/1: 83-125.

2001. La continuidad del cambio lingüístico. Granada: Universidad de Granada. 